\title{
Impaired cognitive performance in premature newborns with two or more surgeries prior to term-equivalent age
}

\author{
Dawn Gano', Sarah K. Andersen², Hannah C. Glass ${ }^{1,3}$, Elizabeth E. Rogers', David V. Glidden4, \\ A. James Barkovich ${ }^{1,3,5}$ and Donna M. Ferriero ${ }^{1,3}$
}

BACKGROUND: Anesthesia in early childhood is associated with adverse neurodevelopmental outcome; however, it is not known if age at exposure affects the risk of adverse outcome. Our objective was to evaluate the association of the number and timing of anesthetic exposures for surgery with cognitive outcome in a cohort of premature newborns.

METHODS: A cohort study of exposure to anesthesia for surgery in premature newborns ( $<33$ wk gestation) prospectively evaluated with neonatal magnetic resonance imaging (MRI) and neurodevelopmental testing at 3-6 y was employed. Exposure to anesthesia for surgery was classified as before term-equivalent age (TEA, $<42 \mathrm{wk}$ postmenstrual age) or after $(\geq 42 \mathrm{wk}$ ). Multivariate regression was performed to analyze the association of composite IQ scores with the number of surgeries before and after TEA.

RESULTS: Among 137 newborns, 25 (18.2\%) had one surgery before TEA and 18 (13.1\%) had $\geq 2$ surgeries. Two or more surgeries before TEA were associated with significantly reduced composite IQ scores at $4.6 \pm 0.6$ y after adjusting for gestational age and illness severity. Neither the number of surgeries after TEA nor sedation for MRI was associated with cognitive outcome.

CONCLUSIONS: More than one surgery prior to TEA is independently associated with impaired cognitive performance in premature newborns.

A $\mathrm{n}$ estimated 6 million pediatric patients require general anesthesia for surgery each year in the United States, including 1.5 million infants (1). A growing body of evidence suggests that general anesthesia has neurotoxic effects on the developing brain (1-14). Several studies have shown that two or more anesthetic exposures prior to $4 \mathrm{y}$ of age are associated with adverse neurodevelopmental outcomes in children (912). However, it is not known if there are windows of selective vulnerability to the effects of anesthesia during critical periods of human brain development (1).

Premature newborns comprise $12 \%$ of all births in the United States (15), and they undergo a remarkable period of brain development by the time they reach term-equivalent age (TEA) (16). Since premature newborns frequently require general anesthesia for surgical complications of prematurity $(6,17,18)$, they constitute a unique population to evaluate how age at exposure to anesthesia for surgery impacts developmental outcome. A large multicenter cohort study (17) has recently shown that major surgery in very low-birth-weight infants was independently associated with an increased risk of death or neurodevelopmental impairment at 18-22 mo' corrected age. However, the number and timing of exposures to anesthesia for surgery was not accounted for in the analysis, and the study population excluded patients with patent ductus arteriosus (PDA) ligation.

Understanding how the timing of exposure to anesthesia for surgery in premature newborns relates to neurodevelopmental outcome has important implications for their clinical care and prognosis. We hypothesized that there would be a decreased cognitive performance in children born prematurely who were exposed to general anesthesia for surgery prior to TEA, independent of illness severity, brain injury on neonatal magnetic resonance imaging (MRI), and exposure to general anesthesia after TEA. To address this hypothesis, we analyzed the association of the number and timing of surgeries to neurodevelopmental outcome in a cohort of premature newborns enrolled in a prospective study of neonatal MRI and evaluated with standardized neurodevelopmental testing at 3-6 y of age.

\section{RESULTS}

The mean gestational age of children enrolled in the cohort was $27.9 \pm 2.4$ wk. Among 137 newborns, 25 (18.3\%) had one surgery prior to TEA and 18 (13.1\%) had $\geq 2$ surgeries (Table 1 ). PDA ligation and laparotomy \pm bowel resection were the most common surgeries before TEA (Table 2). Children who had one or more surgeries prior to TEA were younger and had higher rates of complications of prematurity including prolonged mechanical ventilation, infection, hypotension, PDA, and necrotizing enterocolitis (NEC) (all $P \leq 0.002$; Table 1).

Throughout the follow-up period, 17 children (12.4\%) had one surgery and $11(8 \%)$ had $\geq 2$ surgeries after TEA. Fifteen

\footnotetext{
'Department of Pediatrics, University of California-San Francisco, San Francisco, California; 2Department of Medicine, Queens University, Kingston, Ontario, Canada; ${ }^{3}$ Department of Neurology, University of California, San Francisco, San Francisco, California; ${ }^{4}$ Department of Epidemiology \& Biostatistics, University of California-San Francisco, San Francisco, California; ${ }^{5}$ Department of Radiology, University of California-San Francisco, San Francisco, California. Correspondence: Dawn Gano (Dawn.Gano@ucsf.edu)
} 
Table 1. Clinical characteristics by number of surgeries prior to termequivalent age

\begin{tabular}{|c|c|c|c|c|}
\hline \multirow[b]{2}{*}{ Characteristic $^{\mathrm{a}}$} & \multicolumn{3}{|c|}{$\begin{array}{c}\text { Surgery prior to term-equivalent } \\
\text { age }\end{array}$} & \multirow[b]{2}{*}{$P$ value ${ }^{b}$} \\
\hline & $\begin{array}{l}\text { None } \\
(n=94)\end{array}$ & $\begin{array}{c}1 \\
(n=25)\end{array}$ & $\begin{array}{c}\geq 2 \\
(n=18)\end{array}$ & \\
\hline $\begin{array}{l}\text { Gestational } \\
\text { age (weeks) }\end{array}$ & $28.6 \pm 2.3$ & $26.7 \pm 1.8$ & $25.8 \pm 1.7$ & 0.001 \\
\hline Male & $40(42.6)$ & $11(44)$ & $11(61.1)$ & 0.20 \\
\hline Prenatal steroids & $80(85.1)$ & $20(80)$ & $14(77.8)$ & 0.60 \\
\hline Magnesium sulfate & $45(47.9)$ & $14(56)$ & $11(61.1)$ & 0.51 \\
\hline $\begin{array}{l}\text { Duration of } \\
\text { ventilation (days) }\end{array}$ & $3(0,15)$ & $30(10,43)$ & $42(17,56)$ & 0.001 \\
\hline Infection & $36(38.3)$ & $14(56)$ & $17(94.4)$ & $<0.001$ \\
\hline Hypotension & $40(42.6)$ & $20(80)$ & $14(77.8)$ & $<0.001$ \\
\hline $\begin{array}{l}\text { Patent ductus } \\
\text { arteriosus }\end{array}$ & $32(34)$ & $15(60)$ & $13(72.2)$ & 0.002 \\
\hline $\begin{array}{l}\text { Necrotizing } \\
\text { enterocolitis }\end{array}$ & $4(4.3)$ & $7(28)$ & $6(33.3)$ & $<0.001$ \\
\hline $\begin{array}{l}\text { Surgery after } \\
\text { term-equivalent age }\end{array}$ & & & & $<0.001$ \\
\hline None & $81(86.2)$ & $22(88)$ & $6(33.3)$ & \\
\hline 1 & $9(9.6)$ & $3(12)$ & $5(27.8)$ & \\
\hline$\geq 2$ & $4(4.3)$ & 0 & $7(38.9)$ & \\
\hline $\begin{array}{l}\text { Number of sedated } \\
\text { MRI scans }\end{array}$ & & & & 0.016 \\
\hline None & $57(60.6)$ & $12(48)$ & $4(22.2)$ & \\
\hline 1 & $26(27.7)$ & $9(36)$ & $7(38.9)$ & \\
\hline$\geq 2$ & $11(11.7)$ & $4(16)$ & $7(38.9)$ & \\
\hline White matter injury & $21(22.3)$ & $5(20)$ & $4(22.2)$ & 1.0 \\
\hline $\begin{array}{l}\text { Intraventricular } \\
\text { hemorrhage }\end{array}$ & & & & 0.17 \\
\hline None & $72(76.6)$ & $17(68)$ & $12(66.7)$ & \\
\hline $\begin{array}{l}\text { Mild (grades } 1 \\
\text { and 2) }\end{array}$ & $20(21.3)$ & $7(28)$ & $4(22.2)$ & \\
\hline $\begin{array}{l}\text { Severe (grades } 3 \\
\text { and 4) }\end{array}$ & $2(2.1)$ & $1(4)$ & $2(11.1)$ & \\
\hline
\end{tabular}

a Mean \pm SD, number (\%), or median (interquartile range). ${ }^{b} P$ value corresponds to Fisher's exact test for categorical variables and Kruskal-Wallis test for continuous variables.

children $(15 / 43,34.9 \%)$ who required surgery prior to TEA had surgery after TEA, and 13 children (13/94, 13.8\%) who did not require surgery prior to TEA had surgery after TEA (Table 1). Diverse types of surgeries were required after TEA (Table 2), most commonly hernia repair and laparotomy.

Anesthetic agents varied widely across subjects before and after TEA (Table 3). Intraoperative complications were documented in five subjects with surgery prior to TEA (Table 2). There were no intraoperative complications documented in surgeries after TEA. Dopamine was required for intraoperative maintenance of blood pressure in eight subjects prior to TEA and in each case was initiated before surgery.
Table 2. Types of surgeries before and after TEA

\begin{tabular}{lcc}
\hline & \multicolumn{2}{c}{ Timing of surgery } \\
\cline { 2 - 3 } & $\begin{array}{c}\text { Prior to TEA } \\
(n=43)\end{array}$ & $\begin{array}{c}\text { After TEA } \\
(n=28)\end{array}$ \\
\hline Total number of surgeries & 80 & 58 \\
Types of surgeries & - & - \\
Abdominal & $17(21.3)$ & $8(13.8)$ \\
$\quad$ Laparotomy \pm resection & $10(12.5)$ & $12(20.7)$ \\
Hernia repair & $2(2.5)$ & - \\
$\quad$ Gastrostomy tube & $11(13.8)$ & $3(5.2)$ \\
Ophthalmologic & $25(31.3)$ & - \\
Patent ductus arteriosus ligation & $6(7.5)$ & $4(6.9)$ \\
Ventricular shunt, reservoir, or revision & $1(1.3)$ & $9(15.5)$ \\
Urological & $8(10)$ & $22(37.9)$ \\
Othera,b & $5(6.3)$ & 0 \\
\hline Intraoperative complication ${ }^{c}$ & &
\end{tabular}

Data presented as number (\% of total number of surgeries).

TEA, term-equivalent age.

aBefore TEA: Broviac line insertion or removal $(n=3)$, tracheostomy $(n=1)$,

biopsy $(n=3)$, diaphragmatic plication $(n=1)$. ${ }^{b}$ After TEA: scopes (bronchoscopy, laryngoscopy, endoscopy, sigmoidoscopy) (n=5), Broviac line insertion or removal $(n=2)$, toe duplication $(n=1)$, dental $(n=5)$, hemangioma removal $(n=1)$, ear/ nose/throat $(n=7)$, thoracoscopic drainage $(n=1)$. c Documented intraoperative complications included: self-limited oxygen desaturation during lung retraction for PDA ligation ( $n=1)$, hypotensive episode without intervention $(n=1)$, bradycardia treated with atropine $(n=1)$, desaturation and bradycardia treated with epinephrine and chest compressions $(n=1)$, apnea and bradycardia post-extubation treated with naloxone $(n=1)$.

Table 3. Type of anesthetic exposure and duration of exposure before and after term-equivalent age

\begin{tabular}{lcc}
\hline & \multicolumn{2}{c}{ Timing of surgery } \\
\cline { 2 - 3 } & $\begin{array}{l}\text { Prior to TEA } \\
(n=43)\end{array}$ & $\begin{array}{c}\text { After TEA } \\
(n=28)\end{array}$ \\
\hline $\begin{array}{l}\text { Median duration, minutes (IQR) } \\
\text { Inhalational anesthesia }\end{array}$ & $80(60,110)$ & $90(73,128)$ \\
Sevoflurane & $7(16.3)$ & $20(71.4)$ \\
Isoflurane & $1(2.3)$ & $5(17.9)$ \\
Halothane & $3(7.0)$ & $2(7.1)$ \\
Nitrous oxide & $5(11.6)$ & $6(21.4)$ \\
Intravenous agents & & \\
Propofol & $2(4.7)$ & $8(28.6)$ \\
Midazolam & $10(23.3)$ & $3(10.7)$ \\
Lorazepam & $5(11.6)$ & 0 \\
Ketamine & 0 & 0 \\
Opioids & & $12(42.9)$ \\
Fentanyl & $22(51.2)$ & $1(3.6)$ \\
Morphine & $9(20.9)$ & \\
\hline
\end{tabular}

TEA, term-equivalent age.

${ }^{a}$ Data presented as number (\%) of subjects exposed to each agent. 
Table 4. Comparison of clinical characteristics by follow-up at 3-6y

\begin{tabular}{|c|c|c|c|}
\hline \multirow[b]{2}{*}{ Characteristic $^{\mathrm{a}}$} & \multicolumn{2}{|c|}{ Follow-up at 3-6y } & \multirow[b]{2}{*}{$P$ value ${ }^{b}$} \\
\hline & $\begin{array}{c}\text { Yes } \\
(n=137)\end{array}$ & $\begin{array}{c}\text { No } \\
(n=116)\end{array}$ & \\
\hline Gestational age (weeks) & $27.9 \pm 2.4$ & $28.5 \pm 2.4$ & 0.070 \\
\hline Male & $62(45.3)$ & $67(57.8)$ & 0.095 \\
\hline Prenatal steroids & $114(83.2)$ & $86(74.1)$ & 0.089 \\
\hline Magnesium sulfate & $70(51.1)$ & $53(45.7)$ & 0.45 \\
\hline Duration of ventilation (days) & $7(1,30)$ & $4(0,19)$ & 0.006 \\
\hline Infection & $67(48.9)$ & $64(55.2)$ & 0.38 \\
\hline Hypotension & $74(54.0)$ & $45(38.8)$ & 0.017 \\
\hline Patent ductus arteriosus & $60(43.8)$ & $37(31.9)$ & 0.069 \\
\hline Necrotizing enterocolitis & $17(12.4)$ & $15(12.9)$ & 1.0 \\
\hline Surgery before term-equivalent age & & & 0.39 \\
\hline None & $94(68.6)$ & $88(75.9)$ & \\
\hline 1 & $25(18.2)$ & $18(15.5)$ & \\
\hline$\geq 2$ & $18(13.1)$ & $10(8.6)$ & \\
\hline Surgery after term-equivalent age & & & 1.0 \\
\hline None & $109(79.6)$ & $92(79.3)$ & \\
\hline 1 & $17(12.4)$ & $14(12.1)$ & \\
\hline$\geq 2$ & $11(8.0)$ & $10(8.6)$ & \\
\hline Sedation for MRI & $64(46.7)$ & $48(41.4)$ & 0.38 \\
\hline White matter injury & $30(21.9)$ & $22(19)$ & 0.95 \\
\hline Intraventricular hemorrhage & & & 0.085 \\
\hline None & $101(73.7)$ & $69(59.5)$ & \\
\hline Mild (grade 1 or 2 ) & $31(22.6)$ & $34(29.3)$ & \\
\hline Severe (grade 3 or 4 ) & $5(3.6)$ & $13(11.2)$ & \\
\hline
\end{tabular}

aMean \pm SD, number (\%), or median (interquartile range). ${ }^{b} P$ value corresponds to Fisher's exact test for categorical variables and Kruskal-Wallis test for continuous variables.

\section{Neurodevelopmental Outcome}

The mean age at follow-up was $4.6 \pm 0.6 \mathrm{y}$. There was no differential loss to follow-up by the number of surgeries required before and after TEA (both $\mathrm{P} \geq 0.39$; Table 4). Testing with the Wechsler Preschool and Primary Scale of Intelligence, 3rd edition (WPPSI-III), was performed in 128/137 children (93.4\%) with follow-up between 3 and 6 y of age. The mean full-scale IQ was $98.7 \pm 15.8$, performance IQ $97.4 \pm 16.0$, and verbal IQ $99.5 \pm 14.5$ points (Figure 1 ). In a simplified model evaluating the relationship of the number and timing of surgeries with composite IQ scores, $\geq 1$ surgery prior to TEA was associated with decreased full-scale IQ, and $\geq 2$ surgeries prior to TEA were associated with decreased performance and verbal IQ (all $P \leq 0.004$; Table 5).

The relationship between the number and timing of surgeries and composite IQ scores was also evaluated in a multivariable model, adjusting for the effects of gestational age, prenatal steroids, hypotension, PDA, NEC, number of infections, duration of mechanical ventilation, white matter injury, and number of sedated MRI scans (Table 6). Two or more surgeries prior to TEA were independently associated with reduced full-scale IQ

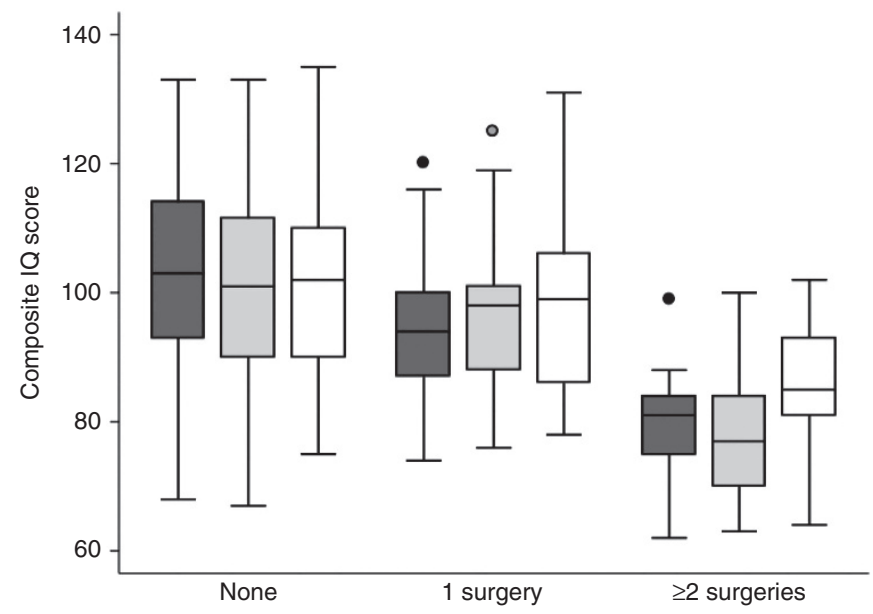

Figure 1. Composite IQ scores by number of surgeries prior to term-equivalent age. Box plot demonstrating composite full-scale (dark gray), performance (light gray), and verbal (white) IQ scores in children by the number of surgeries prior to term-equivalent age (TEA). Circles represent outliers.

(mean difference: -20.3 points; $95 \%$ confidence interval (CI): -32.6 to $-10.1 ; P=0.001$ ), performance IQ (mean difference: -22.7 points; $95 \% \mathrm{CI}:-34.4$ to $-12.6 ; P<0.001)$ and verbal IQ (mean difference: -12.7 points; $95 \% \mathrm{CI}$ : -21.7 to -0.4 ; $P=0.031$ ). In a sensitivity analysis excluding subjects with documented intraoperative complications or intraoperative treatment with dopamine for maintenance of blood pressure, the results were unchanged.

There were 22 children who had an abnormal neurological examination, of whom, 12 had surgery before TEA and 3 had surgery after TEA. Children who had surgery prior to TEA were more likely to have an abnormal neurological examination (risk ratio: 2.62; 95\% CI: $1.23-5.60 ; P=0.011$ ). After adjustment for clinical characteristics associated with surgery prior to TEA, white matter injury, and the number of sedated MRI scans, there was no relationship between the number and timing of surgeries and an abnormal neurological examination (all $\mathrm{P} \geq 0.37$ ).

\section{Sedation for MRI and Cognitive Outcome}

Sedation for neonatal MRI was more common in newborns who had surgery prior to TEA compared with those who did not (risk ratio: 1.65 ; 95\% CI: $1.19-2.31 ; P=0.0051$ ). There was no relationship between the number of sedated MRI scans and composite IQ scores in the multivariable model, adjusting for the number and timing of surgeries, clinical characteristics associated with surgery prior to TEA, and white matter injury (Table 7). There was no effect modification of the association between the number and timing of surgeries and composite IQ scores by sedation for MRI in this model (all $P \geq 0.20$ ).

\section{DISCUSSION}

Two or more surgeries prior to term-equivalent age are associated with decreased composite IQ scores at $4.6 \mathrm{y}$ in this cohort of children born prematurely. Children who required surgery prior to TEA were born at a younger gestational 
Table 5. Association of the number and timing of surgeries with composite IQ scores

\begin{tabular}{lcc}
\hline & $\begin{array}{c}\text { Adjusted mean } \\
\text { difference }(95 \% \mathrm{Cl})\end{array}$ & $P$ value \\
\hline Full-scale IQ & Ref & \\
Number of surgeries before term-equivalent age & - \\
None & $-8.3(-17.6$ to -2.3$)$ & 0.004 \\
1 & $-22.7(-28.0$ to -16.6$)$ & $<0.001$
\end{tabular}

Number of surgeries after term-equivalent age

$\begin{array}{lcc}\text { None } & \text { Ref } & - \\ 1 & -4.4(-13.0 \text { to } 8.2) & 0.39 \\ \geq 2 & -8.4(-24.7 \text { to } 5.5) & 0.25\end{array}$

Performance IQ

Number of surgeries before term-equivalent age

\begin{tabular}{llc} 
None & \multicolumn{1}{c}{ Ref } & - \\
1 & $-4.3(-11.2$ to 4.2$)$ & 0.18 \\
$\geq 2$ & $-22.2(-29.7$ to -17.3$)$ & $<0.001$
\end{tabular}

Number of surgeries after term-equivalent age

\begin{tabular}{llc} 
None & \multicolumn{1}{c}{ Ref } & - \\
1 & $-0.7(-15.5$ to 8.1$)$ & 0.89 \\
$\geq 2$ & $-9.2(-22.3$ to 10.5$)$ & 0.27
\end{tabular}

Verbal IQ

Number of surgeries before term-equivalent age

\begin{tabular}{lcc} 
None & \multicolumn{1}{c}{ Ref } & - \\
1 & $-3.9(-10.5$ to 1.7$)$ & 0.24 \\
$\geq 2$ & $-15.7(-22.1$ to -8.8$)$ & $<0.001$
\end{tabular}

Number of surgeries after term-equivalent age

\begin{tabular}{lcc} 
None & Ref & - \\
1 & $-3.4(-14.1$ to 9.6$)$ & 0.55 \\
$\geq 2$ & $-2.3(-11.0$ to 9.1$)$ & 0.76 \\
\hline
\end{tabular}

$\mathrm{Cl}$, confidence interval.

aGeneralized estimating equation of the mean difference in composite IQ scores associated with the number of surgeries before and after term-equivalent age.

age, and had higher rates of complications of prematurity. After adjustment for multiple confounding variables, including gestational age and white matter injury, $\geq 2$ surgeries prior to TEA were associated with decreased composite IQ scores. The number of surgeries performed after TEA was not associated with cognitive outcome. Surgery prior to TEA was also associated with an increased risk of an abnormal neurological examination on follow-up; however, this was not significant after adjustment for confounding variables. These data indicate that $\geq 2$ surgeries before TEA are independently associated with impaired cognitive development in premature newborns.
Table 6. $\geq 2$ surgeries before term-equivalent age are independently associated with reduced composite IQ scores

\begin{tabular}{lcc}
\hline & $\begin{array}{c}\text { Adjusted mean } \\
\text { difference }(95 \% \mathrm{Cl})\end{array}$ & $P$ value \\
\hline $\begin{array}{l}\text { Full-scale IQ } \\
\text { Number of surgeries before term-equivalent age }\end{array}$ & \multicolumn{1}{c}{ Ref } & - \\
None & $-6.0(-13.0$ to 1.6$)$ & 0.088 \\
1 & $-20.3(-32.6$ to -10.1$)$ & 0.001 \\
$\geq 2$ & Ref & - \\
Number of surgeries after term-equivalent age & \\
None & $0.7(-15.3$ to 10.9$)$ & 0.90 \\
1 & $8.6(-5.4$ to 17.1$)$ & 0.15
\end{tabular}

Performance IQ

Number of surgeries before term-equivalent age

\begin{tabular}{llc} 
None & \multicolumn{1}{c}{ Ref } & - \\
1 & $-3.0(-10.9$ to 2.2$)$ & 0.43 \\
$\geq 2$ & $-22.7(-34.4$ to -12.6$)$ & $<0.001$
\end{tabular}

Number of surgeries after term-equivalent age

$\begin{array}{lcc}\text { None } & \text { Ref } & - \\ 1 & 6.0(-6.7 \text { to } 17.1) & 0.34 \\ \geq 2 & 8.8(-1.3 \text { to } 18.9) & 0.16\end{array}$

Verbal IQ

Number of surgeries before term-equivalent age

\begin{tabular}{lcc} 
None & \multicolumn{1}{c}{ Ref } & - \\
1 & $-0.02(-6.6$ to 11.2$)$ & 0.997 \\
$\geq 2$ & $-12.7(-21.7$ to -0.4$)$ & 0.031
\end{tabular}

Number of surgeries after term-equivalent age

\begin{tabular}{lcc} 
None & Ref & - \\
1 & $1.9(-17.3$ to 10.7$)$ & 0.75 \\
$\geq 2$ & $11.7(-5.9$ to 24.6$)$ & 0.15 \\
\hline
\end{tabular}

$\mathrm{Cl}$, confidence interval; NEC, necrotizing enterocolitis; PDA, patent ductus arteriosus. aGeneralized estimating equation model adjusted for gestational age, duration of mechanical ventilation, number of infections, PDA, NEC, white matter injury, number of sedated scans, as well as the number of surgeries before and after term-equivalent age.

It is difficult to separate the potential deleterious effects of general anesthesia from the effects of surgery such as systemic inflammation or physiologic derangements that may take place intraoperatively, including impaired cerebral perfusion (1). Moreover, the requirement for surgery in our cohort was associated with multiple markers of systemic illness. We attempted to estimate the direct effects of surgery on neurodevelopment by adjusting for several covariates associated with surgery prior to TEA using bootstrapping methodology; however, we cannot exclude residual confounding in our results. In a sensitivity analysis excluding newborns with documented 
intraoperative complications, or intraoperative treatment with a vasopressor for maintenance of blood pressure, the interpretation of the models was similar. The demonstrated association of $\geq 2$ surgeries prior to TEA and impaired cognitive performance highlights that this subset of premature newborns is at high risk for cognitive deficits but does not necessarily represent a causal relationship between anesthesia exposure and abnormal cognitive outcome.

Our findings are consistent with a growing body of evidence that suggests exposure to general anesthesia has adverse effects on the developing brain $(1-14,17,18)$. Studies in infant animal models have demonstrated a developmental vulnerability of the brain to the neurotoxic effects of commonly used anesthetic agents, such as the inhalational anesthetics $(1-5,13,14)$. In addition to widespread neuronal apoptosis, early exposure to general anesthesia and sedation in infant animal models is associated with long-term cognitive and behavioral effects $(1-5,13,19)$. The brain appears to be more susceptible to the neurotoxic effects of anesthesia during periods of rapid growth and development, as well as peak synaptogenesis $(1-4,13,14)$.

The potential neurotoxic effects of general anesthesia on brain development have garnered attention from the US Food and Drug Administration, as well as the media (20-22). Prospective studies and randomized trials are currently underway to further address this public health issue and inform clinical practice $(1,22)$. The question of whether exposure to general anesthesia impacts brain development is a source of great concern to parents of infants and children who require anesthesia for surgery, as well as those who require sedation for MRI.

We found no significant association between the number of sedated MRI scans and cognitive outcome at $4.6 \mathrm{y}$ in our cohort after adjustment for confounding variables, including the number and timing of surgeries. Our institutional practice includes sedation with morphine and/or pentobarbital for neonatal MRI scans if newborns are actively moving during the scan. Since sedation for MRI limits motion and enables higher quality MR images to be obtained, our results are reassuring that the practice of sedation for scanning should not be precluded by concerns of sedation-related effects on cognitive outcome.

The results of our study are concordant with a recent meta-analysis (12) of seven studies, which demonstrated an increased risk of learning and behavioral disorders associated with an increased number of anesthetic exposures prior to 4 y. Age at exposure, defined in yearly intervals, was not associated with an increased risk of impaired neurodevelopment. However, only three studies in the meta-analysis $(10,11,23)$ reported inclusion of children born less than $33 \mathrm{wk}$ gestation and none of the studies in the meta-analysis specifically evaluated the impact of anesthesia exposure for surgery prior to TEA. It is not known if there exists a critical period of selective vulnerability to the effects of anesthesia and surgery during human brain development (1). Our results indicate that $\geq 2$ exposures to anesthesia for surgery prior to TEA are associated with significantly reduced cognitive performance, whereas
Table 7. Sedation for MRI is not associated with cognitive performance

\begin{tabular}{lcc}
\hline \multicolumn{1}{c}{$\begin{array}{c}\text { Adjusted mean } \\
\text { difference }(95 \% \mathrm{Cl})\end{array}$} & $P$ value \\
\hline $\begin{array}{l}\text { Full-scale IQ } \\
\text { Number of sedated MRI scans } \\
\text { None }\end{array}$ & Ref & - \\
1 & $0.4(-4.8$ to 6.7$)$ & 0.90 \\
$\geq 2$ & $-2.7(-8.3$ to 6.8$)$ & 0.44 \\
Performance IQ & & \\
Number of sedated MRI scans & Ref & - \\
None & $1.0(-6.5$ to 8.4$)$ & 0.79 \\
1 & $-3.8(-11.4$ to 3.8$)$ & 0.38 \\
$\geq 2$ & &
\end{tabular}

Verbal IQ

Number of sedated MRI scans

None

1

$\geq 2$

$\begin{array}{cc}\text { Ref } & - \\ 0.3(-6.5 \text { to } 7.5) & 0.94 \\ -0.6(-6.4 \text { to } 7.0) & 0.87\end{array}$

$\mathrm{Cl}$, confidence interval; MRI, magnetic resonance imaging; NEC, necrotizing enterocolitis; PDA, patent ductus arteriosus.

a Generalized estimating equation model adjusted for gestational age, duration of mechanical ventilation, number of infections, PDA, NEC, white matter injury, number of sedated scans, as well as the number of surgeries before and after term-equivalent age.

exposure after TEA is not associated with cognitive performance in our cohort. Premature newborns undergo a remarkably intricate period of brain growth and development as they approach TEA, characterized by cortical sulcation, synaptogenesis, myelination, and cerebellar growth $(16,24,25)$. This period of rapid change is also characterized by maturation of neurons and axonal development, as well as a peak abundance of microglia (25). Our results suggest that the impact of anesthetic exposure and surgery during this period of human brain development merits further study.

Children that required neonatal surgery have previously been reported to have an increased risk of neurosensory impairment (26); worse academic performance in adolescence compared to controls (27); and impaired neurodevelopment in infancy $(17,18)$. None of these studies accounted for surgeries required after the neonatal period. A large multicenter cohort study (17) has recently shown that major surgery in very-lowbirth-weight infants was independently associated with an increased risk of death or neurodevelopmental impairment at 18-22 mo' corrected age. However, the number and timing of exposures to anesthesia for surgery was not evaluated, the study population excluded newborns that required PDA ligation, and only severe cystic white matter injury detected by ultrasound was adjusted for in the analysis. Filan et al. (18) found that very preterm infants undergoing surgery had reduced cognitive performance at $2 \mathrm{y}$ of corrected age compared with infants who did not have surgery, but in contrast with our results, this difference was not significant after adjustment for confounding variables. In addition, infants in the 
surgical group had reduced deep nuclear gray matter volume at TEA (18), which may indicate regional vulnerability to the effects of anesthesia and/or surgery.

Many of the previous studies that reported an association between anesthesia and adverse neurodevelopment included children with underlying conditions that may contribute to poor developmental outcomes. The well-defined study population and exclusion of newborns with evidence of genetic syndromes and congenital malformations, such as malformations of brain development, strengthens our study. Our study is also strengthened by the inclusion of neonatal MRI findings, and adjustment for noncystic white matter injury, which is more prevalent than cystic white matter injury in premature newborns (28), and best detected by MRI (29). In addition, detailed review of the medical records enabled us to account for the potential effects of perioperative and intraoperative complications. Due to the size of the study population and the nature of multiple co-exposures in several subjects, we were not powered to evaluate associations of specific medications with neurodevelopmental outcome or dose-dependent effects. Although developmental followup was only available for a subset of the primary cohort evaluated with neonatal MRI, there was no differential loss to follow-up by the number and timing of surgeries required. The number of surgeries performed after TEA may have been underestimated if the surgery was not performed at our center; however, surgical history was obtained at each follow-up visit. Detailed socioeconomic data were not available for this analysis.

There is an urgent need to identify modifiable risk factors for adverse neurodevelopment in premature newborns. Our study showed a strong association between more than one exposure to anesthesia for surgery prior to term-equivalent age and decreased cognitive performance at $4.6 \mathrm{y}$, and no association between MRI with sedation and any outcome measure. Further study is needed to understand how general anesthesia and surgery affect brain growth and development, particularly in premature newborns. Future studies should focus on the development and implementation of neuroprotective strategies for preterm newborns undergoing surgery.

\section{METHODS \\ Study Design}

This is a cohort study of exposure to general anesthesia for surgery among premature newborns prospectively studied with neonatal MRI and neurodevelopmental testing at 3-6 y. The primary outcome measure was cognitive performance on the Wechsler Preschool and Primary Scale of Intelligence, 3rd edition. The secondary outcome measure was an abnormal neurological examination.

\section{Study Subjects}

The cohort comprised 137 premature newborns <33 wks' gestation admitted to the intensive care nursery at the University of California, San Francisco (UCSF) from January 1998 to April 2009 who were enrolled in a prospective study of neonatal MRI. Exclusion criteria include clinical evidence of a congenital malformation or syndrome, congenital infection, or clinical status too unstable for transport to MRI. Parental consent was obtained following a protocol approved by the UCSF Committee on Human Research.
Clinical data were extracted from the medical records by two investigators (D.G. and S.K.A.) blinded to neurodevelopmental outcome. The total number, timing, and type of surgery were obtained from the medical records. Surgery was defined as a procedure requiring general anesthesia in the operating room, or interventional radiology suite. Exposure to inhalational anesthesia (sevoflurane, isoflurane, halothane, nitrous oxide), intravenous agents (propofol, ketamine, midazolam, pentobarbital), and opioids (fentanyl, morphine) was determined by reviewing the anesthetic records from each surgery. Doses and concentrations of anesthetic exposures were not consistently available. The timing of surgery was classified as prior to TEA ( $<42 \mathrm{wk}$ postmenstrual age) or after TEA ( $\geq 42 \mathrm{wk}$ postmenstrual age). The occurrence of documented intraoperative complications was also obtained.

Additional covariates prospectively collected include gestational age, prenatal steroids and magnesium sulfate, infection, duration of mechanical ventilation (days), hypotension requiring medical intervention, PDA, NEC, and hypoglycemia. Newborns with culturepositive sepsis, clinical signs of sepsis with negative blood culture, or meningitis were classified as having infection. Newborns with clinical signs and symptoms of NEC and evidence of pneumatosis intestinalis on X-ray were classified as having NEC.

MRI scans were obtained as soon after birth as newborns were clinically stable and near TEA. A custom MR-compatible incubator was used to provide a quiet, well-monitored environment for newborns, minimizing patient movement and improving the signalto-noise ratio (30). Scans were performed without sedation if possible. The sedation protocol for neonatal MRI in our cohort includes intravenous morphine and/or pentobarbital as needed to reduce patient movement. General anesthesia is not used for neonatal MRI at our institution. There were no adverse events due to sedation for neonatal MRI. The majority of all sedated MRI scans were performed prior to TEA (84/86). A single pediatric neuroradiologist (A.J.B.) evaluated all MRI scans blinded to the clinical history (other than postmenstrual age at the time of scan). The severity of white matter injury on $\mathrm{T}_{1}$-weighted MRI was scored according to previously published criteria (29). White matter injury was dichotomized as present or absent. Intraventricular hemorrhage was classified using the Papile grading system (31). The highest intraventricular hemorrhage score from both scans was included in the analysis.

\section{Neurodevelopmental Outcome}

After hospital discharge, children had serial neurodevelopmental assessments prior to $3 \mathrm{y}$ of age in the UCSF Intensive Care Nursery Follow-Up Program as part of their clinical care. Children enrolled in our neonatal MRI research cohort were invited for further followup between 3 and 6 y of age, which was obtained in 137 children (54.2\%). Follow-up visits between 3 and 6 y included a standardized neurological examination and neurodevelopmental assessment, with the examiner blinded to the medical history including exposure to anesthesia. The neurological examination was summarized using a validated neuromotor score (0-6), which scores abnormalities in cranial nerve function, muscle tone, strength, and deep tendon reflexes $(32,33)$. Subjects with a neuromotor score $\geq 1$ were classified as having an abnormal neurological examination. Neurodevelopmental assessment consisted of evaluation by a developmental psychologist using the WPPSI-III (The Psychological Corporation, 2002), which is a standardized and validated scale. We recorded full-scale IQ, verbal IQ, and performance IQ. The mean of each composite score of the WPPSI is 100 points and the SD is 15.

\section{Analysis}

Statistical analysis was performed using Stata 13 (Stata Corporation, College Station, TX). Clinical characteristics of subjects who required surgery prior to TEA and those who did not were compared using descriptive statistics. Categorical variables were compared using Fisher's exact test, and continuous variables were compared using Kruskal-Wallis test. A generalized estimating equation was used to assess the relationship between the number and timing of surgeries with composite IQ scores on the WPPSI-III. In the adjusted model, we included variables associated 
with surgery prior to TEA $(P<0.1)$, white matter injury on neonatal MRI, and the total number of sedated MRI scans. Bootstrap analysis was used to estimate bias-corrected SEs and 95\% CI. Bootstrapping entails random resampling of the study population to obtain the empirical distribution and determine the standard errors with greater accuracy when the sampling distribution of the statistic of interest is unlikely to be normal (34). Effect modification of the relationship between the timing and number of surgeries and composite IQ scores by the number of sedated MRI scans was also evaluated in this model. A generalized estimating equation was used to evaluate the relationship of the number and timing of surgeries with an abnormal neurological examination, adjusting for variables associated with surgery prior to TEA $(P<0.1)$, white matter injury on neonatal MRI, and the total number of sedated MRI scans. Statistical significance was set at an $\alpha$ of 0.05 .

\section{STATEMENT OF FINANCIAL SUPPORT}

This research is supported by the National Institutes of Health (Bethesda, MD) grants NS35902, NS40227, NS046432, and Thrasher Research Fund (Salt Lake City, UT) Early Career Award 12475.

Disclosure: None.

\section{REFERENCES}

1. Sun L. Early childhood general anaesthesia exposure and neurocognitive development. Br J Anaesth 2010;105:Suppl 1:161-8.

2. Jevtovic-Todorovic V, Hartman RE, Izumi Y, et al. Early exposure to common anesthetic agents causes widespread neurodegeneration in the developing rat brain and persistent learning deficits. J Neurosci 2003;23:876-82.

3. Paule MG, Li M, Allen RR, et al. Ketamine anesthesia during the first week of life can cause long-lasting cognitive deficits in rhesus monkeys. Neurotoxicol Teratol 2011;33:220-30.

4. Deng M, Hofacer RD, Jiang C, et al. Brain regional vulnerability to anaesthesia-induced neuroapoptosis shifts with age at exposure and extends into adulthood for some regions. Br J Anaesth 2014;113:443-51.

5. Davidson AJ. Anesthesia and neurotoxicity to the developing brain: the clinical relevance. Paediatr Anaesth 2011;21:716-21.

6. Walker K, Holland AJ, Winlaw D, Sherwood M, Badawi N. Neurodevelopmental outcomes and surgery in neonates. J Paediatr Child Health 2006;42:749-51.

7. DiMaggio C, Sun LS, Kakavouli A, Byrne MW, Li G. A retrospective cohort study of the association of anesthesia and hernia repair surgery with behavioral and developmental disorders in young children. J Neurosurg Anesthesiol 2009;21:286-91.

8. Ing C, DiMaggio C, Whitehouse A, et al. Long-term differences in language and cognitive function after childhood exposure to anesthesia. Pediatrics 2012;130:e476-85.

9. DiMaggio C, Sun LS, Li G. Early childhood exposure to anesthesia and risk of developmental and behavioral disorders in a sibling birth cohort. Anesth Analg 2011;113:1143-51.

10. Wilder RT, Flick RP, Sprung J, et al. Early exposure to anesthesia and learning disabilities in a population-based birth cohort. Anesthesiology 2009;110:796-804.

11. Flick RP, Katusic SK, Colligan RC, et al. Cognitive and behavioral outcomes after early exposure to anesthesia and surgery. Pediatrics 2011;128: e1053-61.

12. Wang X, Xu Z, Miao CH. Current clinical evidence on the effect of general anesthesia on neurodevelopment in children: an updated systematic review with meta-regression. PLoS One 2014;9:e85760.

13. Stratmann G, Sall JW, May LD, et al. Isoflurane differentially affects neurogenesis and long-term neurocognitive function in 60-day-old and 7day-old rats. Anesthesiology 2009;110:834-48.
14. Rizzi S, Ori C, Jevtovic-Todorovic V. Timing versus duration: determinants of anesthesia-induced developmental apoptosis in the young mammalian brain. Ann NY Acad Sci 2010;1199:43-51.

15. Hamilton BE, Martin JA, Ventura SJ. Births: preliminary data for 2012. Natl Vital Stat Rep 2013;62:1-20.

16. Volpe JJ. Neurology of the Newborn, 5th edn. Philadelphia, PA: WB Saunders Company, 2008.

17. Morriss FH Jr, Saha S, Bell EF, et al.; Eunice Kennedy Shriver National Institute of Child Health and Human Development Neonatal Research Network. Surgery and neurodevelopmental outcome of very low-birthweight infants. JAMA Pediatr 2014;168:746-54.

18. Filan PM, Hunt RW, Anderson PJ, Doyle LW, Inder TE. Neurologic outcomes in very preterm infants undergoing surgery. J Pediatr 2012;160: 409-14.

19. Stratmann G, Lee J, Sall JW, et al. Effect of general anesthesia in infancy on long-term recognition memory in humans and rats. Neuropsychopharmacology 2014;39:2275-87.

20. Rappaport B, Mellon RD, Simone A, Woodcock J. Defining safe use of anesthesia in children. N Engl J Med 2011;364:1387-90.

21. Glass NL, Malviya S. Anesthesia in children-limitations of the data on neurotoxicity. N Engl J Med 2011;364:1466-7.

22. Nemergut ME, Aganga D, Flick RP. Anesthetic neurotoxicity: what to tell the parents? Paediatr Anaesth 2014;24:120-6.

23. Hansen TG, Pedersen JK, Henneberg SW, et al. Academic performance in adolescence after inguinal hernia repair in infancy: a nationwide cohort study. Anesthesiology 2011;114:1076-85.

24. Semple BD, Blomgren K, Gimlin K, Ferriero DM, Noble-Haeusslein LJ. Brain development in rodents and humans: identifying benchmarks of maturation and vulnerability to injury across species. Prog Neurobiol 2013;106-107:1-16.

25. Volpe JJ. Brain injury in premature infants: a complex amalgam of destructive and developmental disturbances. Lancet Neurol 2009;8:110-24.

26. Kabra NS, Schmidt B, Roberts RS, Doyle LW, Papile L, Fanaroff A; Trial of Indomethacin Prophylaxis in Preterms Investigators. Neurosensory impairment after surgical closure of patent ductus arteriosus in extremely low birth weight infants: results from the Trial of Indomethacin Prophylaxis in Preterms. J Pediatr 2007;150:229-34, 234.e1.

27. Ludman L, Spitz L, Wade A. Educational attainments in early adolescence of infants who required major neonatal surgery. J Pediatr Surg 2001;36:858-62.

28. Gano D, Andersen SK, Partridge JC, et al. Diminished white matter injury over time in a cohort of premature newborns. J Pediatr 2015;166:39-43.

29. Miller SP, Cozzio CC, Goldstein RB, et al. Comparing the diagnosis of white matter injury in premature newborns with serial MR imaging and transfontanel ultrasonography findings. AJNR Am J Neuroradiol 2003;24:1661-9.

30. Dumoulin CL, Rohling KW, Piel JE, et al. Magnetic resonance imaging compatible neonate incubator. Concepts Magn Reson 2002;15:117-28.

31. Papile LA, Burstein J, Burstein R, Koffler H. Incidence and evolution of subependymal and intraventricular hemorrhage: a study of infants with birth weights less than 1,500 gm. J Pediatr 1978;92:529-34.

32. Miller SP, Latal B, Clark H, et al. Clinical signs predict 30-month neurodevelopmental outcome after neonatal encephalopathy. Am J Obstet Gynecol 2004;190:93-9.

33. Hajnal BL, Sahebkar-Moghaddam F, Barnwell AJ, Barkovich AJ, Ferriero DM. Early prediction of neurologic outcome after perinatal depression. Pediatr Neurol 1999;21:788-93.

34. Vittinghoff E, Glidden DV, Shiboski SC, McCulloch CE. Regression Methods in Biostatistics, 2nd edn. New York, NY: Spring Science + Business Media, 2012. 